\title{
Radiation hardness and stability of optical coupling materials for Bellell electromagnetic calorimeter
}

\author{
G.Finocchiaro, * \\ ${ }^{1}$ Laboratori Nazionali di Frascati dell'INFN \\ Via E. Fermi, 40 - 00044 Frascati, Roma (Italy) \\ E-mail: giuseppe.finocchiaro@lnf.infn.it
}

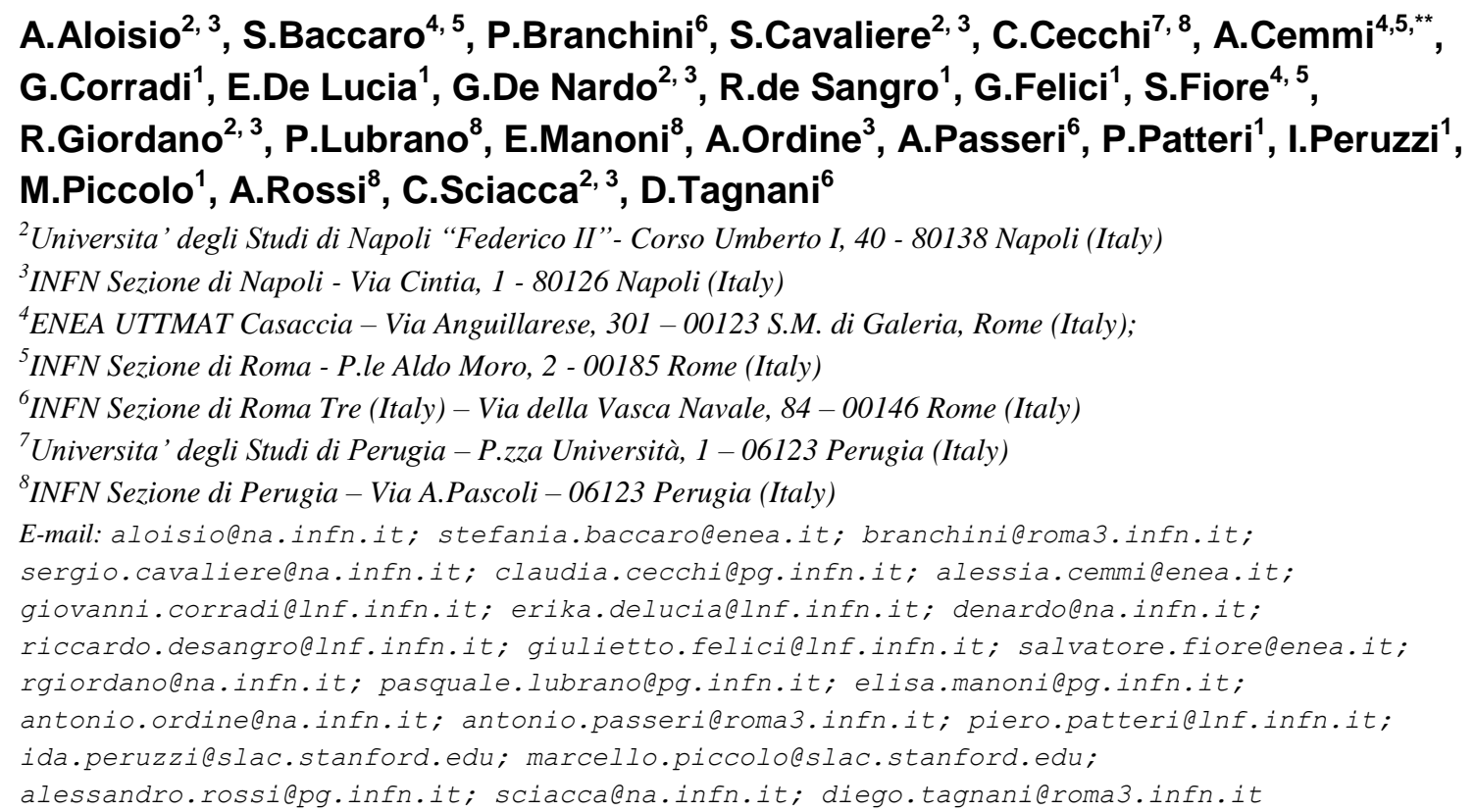

Optical coupling materials are usually applied to ensure good optical matching in the APDscintillating crystal detection system. High transparency at the scintillating emission wavelength and material stability under irradiation are recommended requirements. In this work, silicon optical grease (BC630) and two-component epoxy resins (Epo-tek 301-2FL and Epo-tek 305), to be employed in the electromagnetic calorimeter of the BelleII experiment at the Super-KEKB collider, were investigated to establish their radiation hardness and stability under gamma and neutron irradiation. Optical transmittance measurements were performed in the UV-VIS range, paying particular attention to the materials behavior in the range around $315 \mathrm{~nm}$ (CsI scintillating emission wavelength).

Technology and Instrumentation in Particle Physics 2014

2-6 June, 2014

Amsterdam, the Netherlands

\footnotetext{
* Speaker: G.Finocchiaro

** Corresponding author: A.Cemmi
} 


\section{Introduction}

After its successful data taking on the KEKB collider, the Belle detector [1] is going to be upgraded to cope with the improved luminosity and consequent expected higher background of the SuperKEKB collider [2, 3]. The forward endcap CsI:Tl crystals and PIN photodiodes will be replaced with undoped CsI and new photodetectors. The Total Ionizing Dose (TID) foreseen for the Belle II endcap, $100 \mathrm{~Gy}$ for the whole data taking, could induce color center formation both in crystals and in optical coupling materials, thus impairing the light collection and consequently the energy resolution. High transparency at the scintillating emission wavelength and material stability under irradiation are mandatory requirements. Silicon optical grease BC630 [4] and two-component epoxy resins, Epo-tek 301-2FL and Epo- tek 305 [5], are investigated to establish their radiation hardness and stability under gamma and neutron irradiation, at Calliope and Tapiro facilities of ENEA (Italy) [6].

\section{Experimental}

Silicone optical grease BC630 (Saint-Gobain Crystals, USA) and two-component epoxy resin Epo-tek 301-2FL and Epo-tek 305 (EPOXY TECHNOLOGY Inc., USA), suggested for the application in the UV range, were investigated. Samples were prepared by deposition of different thickness of material on Suprasil 2 Grade B quartz disk (1 inch diameter and $1 \mathrm{~mm}$ thickness, Hereaus Quarzglas, Germany). For the Epo-tek 301-2FL resin, two additional samples are produced, at room temperature and after thermal treatment at $80^{\circ} \mathrm{C}$ for 3 hours, as suggested in the data sheet [5], as self-standing disks of 770 microns thickness. The sample prepared at room temperature was then irradiated by gamma-rays and neutron. Gamma irradiation tests were performed at the Calliope facility (ENEA-Casaccia R.C., Rome, Italy), a pool-type irradiation plant equipped with a ${ }^{60} \mathrm{Co}$ gamma source (mean energy of $1.25 \mathrm{MeV}$ ) in a high volume $\left(7 \times 6 \times 3.9 \mathrm{~m}^{3}\right)$ shielded cell. Absorbed doses up to 530Gy were applied at the dose rate of $5 \mathrm{~Gy}_{\text {air }} / \mathrm{h}$, measured by a Fricke dosimeter All the irradiation tests were carried out in air, at room temperature. Neutron irradiation was carried out at the Tapiro Fast Neutron reactor (ENEA-Casaccia R.C., Rome, Italy, maximum thermal power of $5 \mathrm{~kW}$ and maximum neutron flux at the core center of $2.2 \cdot 10^{12} \mathrm{n} / \mathrm{cm}^{2} \cdot \mathrm{s}$ ) with fluence of $1 \cdot 10^{13} \mathrm{n} / \mathrm{cm}^{2}$. Gamma irradiation effect was evaluated by means of the radiation induced absorption coefficient $\mu$, proportional to the number of the defects induced by ionizing radiation and able to absorb visible light (color centers), defined as: $\mu=1 / d \cdot \ln \left(T_{0} / T\right)$ where $d$ is the sample thickness and $T_{0}$ and $T$ are the transmission spectra before and after irradiation, respectively. Transmission spectra were recorded before and after irradiation, in the range $200-800 \mathrm{~nm}$ by a double-beam Lambda 950 UV-VIS Spectrophotometer (Perkin-Elmer) equipped with an integrating sphere $\left(\% \mathrm{~T}_{\text {error }}= \pm 0.5 \%\right)$. Samples transmittance curves were normalized by the contribution of the quartz substrate.

\section{Results and discussion}

Transmittance curves for different thickness of BC630 silicone grease and of Epo-tek 301 and Epo-tek 305 epoxy-resins were shown in Fig.1. Despite a partial transparency in the UV 
range (below 300nm), Epo-tek 305 presents excessive absorption with the increase of the thickness, making this resin not suitable for the wavelength of BelleII calorimeter interest $(315 \mathrm{~nm})$. Considering the other two materials, a cut-off edge is shown below $315 \mathrm{~nm}$ and very good results in term of transmittance are evident for the silicon grease, regardless of the thickness increase.


Fig.1: Thickness effect on transmittance for BC630silicone grease, Epo-tek 301 and Epo-tek 305 epoxyresins.

To get a complete evaluation of the features of BC630 and Epo-tek 301, their stability was investigated before and after gamma irradiation (13.2Gy) for a period of 3 months. In the first case (data here not shown), the transmittance (\%) values remain practically unchanged for the silicone grease, while the epoxy-resin (prepared at room temperature and at $80^{\circ} \mathrm{C}$ ) presents a general decrease of the transmittance during time (around -5\%/60 days). The radiation induced absorption coefficient of silicone grease and epoxy-resin, recorded immediately after the end of gamma irradiation at $13.2 \mathrm{~Gy}$ absorbed dose and during the following 3 months, is reported in Fig.2.

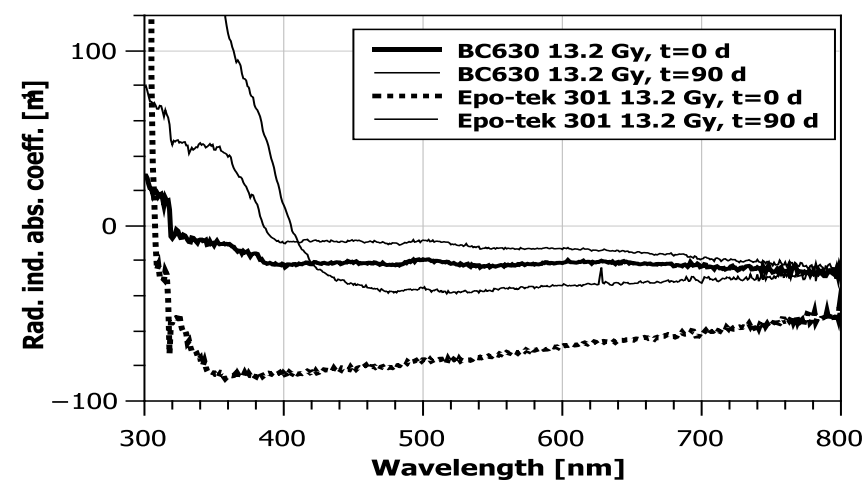

Fig.2: Radiation induced absorption coefficient trend of BC630, Epo-tek 301 after gamma irradiation at 13.2Gy absorbed dose. The behavior up to 90 days after the end of irradiation is also reported. (Statistical

$$
\mu_{\text {error }}= \pm 8 \mathrm{~m}^{-1} \text { ). }
$$

Again, the experimental data demonstrate the highest stability and radiation hardness (especially in the UV range) of BC630. In particular, despite the negligible modification for both the materials immediately after gamma irradiation, 3 months later transmittance (\%) loss for BC630 is around 4\% while for Epo-tek 301 it reaches a value of around 10\%. Based on the outstanding features of the silicone grease, further gamma irradiation was carried out at high absorbed dose (530Gy). As compared in Fig.3a, the increase of the absorbed dose induces, as expected, the creation of a higher number of positively and negatively charged defects and a consequent 
radiation damage enhancement. This behavior, however, is more pronounced for wavelength longer than $320 \mathrm{~nm}$.

Finally, the effect of neutron irradiation is clearly evident from the comparison of the transmittance curves reported in Fig.3b for both the materials investigated. Silicone grease is characterized by a high stability also after neutron irradiation, even showing a partial increase in transmittance below $350 \mathrm{~nm}$, probably due to crystallization phenomena. On the contrary, Epotek 301 transmittance is strongly reduced by neutron irradiation, reaching an unacceptable value $(2.65 \%)$ at $315 \mathrm{~nm}$.
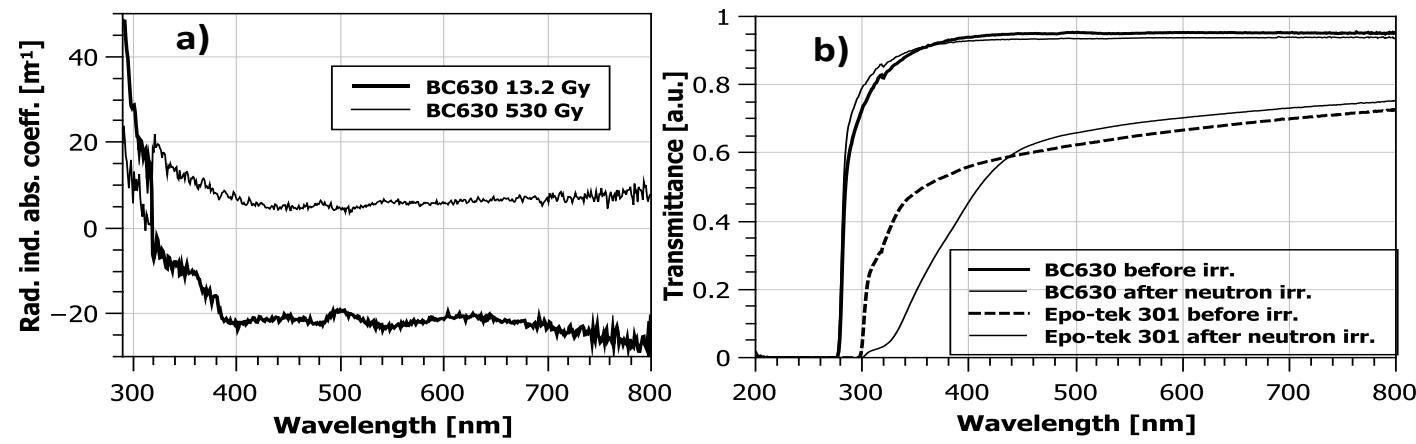

Fig.3: a) Radiation induced absorption coefficient trend of BC630 irradiated at 13.2 Gy and 530 Gy absorbed doses; b) Transmittance spectra before and after neutron irradiation of BC630 (480 micron) and Epo-tek 301 (770 micron).

\section{Conclusions}

Different optical coupling materials to be used in the BelleII electromagnetic calorimeter were investigated in this work. Silicone grease BC630 and two-component epoxy resins (Epo-tek 301 and Epo-tek 305) were analyzed and compared in terms of stability before and after irradiation (gamma, neutron) and of radiation hardness. The thickness layer influence was also studied. The experimental results show that silicone grease BC630 is undoubtedly the most suitable optical coupling material thinking at the application in the UV range $(315 \mathrm{~nm})$.

\section{Acknowledgements}

We want to thank the Tapiro reactor team for their support during setup preparation and for maintaining stable operating conditions during irradiations.

\section{References}

[1] A. Abashian et.al., Nuclear Instruments and Methods A, 479 (2002) 117.

[2] SuperKEKB Letter of Intent, KEK Report 04-4.

[3] Belle II Technical Design Report, arXiv:1011.0352.

[4] http://www.crystals.saint-gobain.com/Assembly Materials.aspx

[5] http://www.epotek.com/site/

[6] S. Baccaro, A.Cecilia, Facilities for radiation hardness qualifications at ENEA-Casaccia, in Proceedings of the $2^{\text {nd }}$ SIRAD Workshop, LNL-INFN (REP) - 203/2004. 